\title{
Eficacia del infliximab en pacientes con síndrome de Behçet portadores de úveo-retinitis grave
}

\author{
G uillermo Merino ${ }^{1,2}$, G onzalo Varas ${ }^{3 a}, G$ onzalo Díaz ${ }^{4 a}$, \\ Miguel Gutiérrez ${ }^{5}$, Loreto Massardo ${ }^{5}$, Daniel Pacheco ${ }^{6}$, \\ Francisco Villarroel ${ }^{7}$ y Miguel Cuchacovich ${ }^{8,9}$. \\ Effectiveness of infliximab in patients \\ with Behçet syndrome and severe \\ uveoretinitis. Report of five cases
}

The efficacy and safety of repeated administration of infliximab was evaluated in five patients (two men, three women) with Behçet syndrome accompanied by severe uveoretinitis. Ocular and extra ocular inflammation was suppressed in all patients during the observation period without any serious adverse reactions. The results in these patients suggests that TNF- $\alpha$ blockade is effective in patients with severe ocular Behçet syndrome (Rev Méd Chile 2006; 134: 875-82).

(Key words: Behçet syndrome; Infliximab; Retinitis; Uveitis)

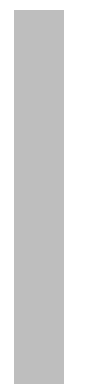

\begin{abstract}
Recibido el 12 de julio, 2005. Aceptado el 10 de enero, 2006.
${ }^{1}$ Departamento de Oftalmología, Clínica Santa María, Santiago, Chile. ${ }^{2}$ Centro Oftalmológico Luis Pasteur, Santiago, Chile. ${ }^{3}$ Facultad de Medicina, Pontificia Universidad Católica de Chile. ${ }^{4}$ Facultad de Medicina, Universidad de Chile. ${ }^{5}$ Departamento de Inmunología Clínica y Reumatología, Facultad de Medicina, Pontificia Universidad Católica de Chile. ${ }^{6}$ Sección de Reumatología, Departamento de Medicina, Hospital San Borja Arriarán, Campus Centro, Facultad de Medicina, Universidad de Chile. ${ }^{7}$ Fundación Oftalmológica Los Andes, Santiago, Chile. ${ }^{8}$ Sección de Reumatología, Departamento de Medicina, Hospital Clínico de la Universidad de Chile, Santiago, Chile. ${ }^{9}$ Sección de Reumatología, Departamento de Medicina, Clínica Las Condes, Santiago, Chile.

aAyudante alumno de Medicina
\end{abstract}

$\mathrm{E}^{1}$ síndrome de Behçet (SB) es una enfermedad inflamatoria crónica, multisistémica, de baja frecuencia en nuestro medio, que se caracteriza por la presencia de úlceras mucosas recurrentes, lesiones cutáneas como eritema nodoso y pseudo-

Correspondencia a: Dr. Miguel Cuchacovich. San Pío X, № 2460, Oficina 607, Providencia, Santiago, Chile. E mail: mcuchaco@yahoo.com. foliculitis, uveítis y tromboflebitis ${ }^{1,2}$. La uveoretinitis (UR) es una complicación mayor del SB que puede llevar a secuelas severas y amaurosis ${ }^{3}$.

Los inmunosupresores (como la ciclosporina A y la azatioprina) son agentes efectivos en el control de los ataques de UR en la mayoría de los pacientes portadores de SB. Sin embargo, en algunos pacientes los inmunosupresores son ineficaces o la aparición de efectos colaterales impide su utilización en dosis adecuadas. 
El factor de necrosis tumoral- $\alpha$ (TNF- $\alpha$ ) es una citoquina proinflamatoria capaz de agravar la uveítis en modelos animales de uveoretinitis experimental autoinmune (UEA) y los anticuerpos monoclonales anti TNF- $\alpha$ son capaces de inhibir la aparición de UEA ${ }^{4,5}$. Ha sido demostrado que clones de linfocitos $\mathrm{T}$ intraoculares y monocitos periféricos de pacientes con SB con UR activa presentan mayor producción de TNF- $\alpha$ que los controles normales ${ }^{6,7}$. Lo anterior sugiere que el TNF- $\alpha$ estaría involucrado en la actividad de la UR en pacientes con SB.

El infliximab es un anticuerpo monoclonal quimérico anti-TNF- $\alpha$ altamente efectivo en el control de los síntomas y de la progresión del daño radiológico en pacientes con artritis reumatoidea ${ }^{8,9}$ y otras enfermedades inmunológicamente mediadas.

A la fecha, hay comunicaciones preliminares de casos clínicos aislados y series pequeñas que sugieren que el infliximab puede ser un agente eficaz en el control de los síntomas clínicos del $\mathrm{SB}^{10-12}$.

El presente estudio tiene por objeto comunicar nuestra experiencia en 5 pacientes con SB activo tratados con infliximab. Todos los pacientes presentaban una severa UR activa antes de iniciar la administración de infliximab. El SB mejoró significativamente en todos ellos sin la aparición de reacciones adversas graves.

\section{PACIENTES Y MÉTODOS}

Cinco pacientes (tres mujeres y dos hombres) fueron incluidos en este estudio. La Tabla 1 muestra las características demográficas de los pacientes. El diagnóstico de SB consideró la presencia de al menos tres criterios clásicos, incluyendo úlceras mucosas recurrentes, severas y UR en todos los pacientes. Todos tenían un PPD menor de $4 \mathrm{~mm}$ y una radiografía de tórax normal al inicio del tratamiento con infliximab.

El infliximab fue administrado en dosis de 3 a $5 \mathrm{mg} / \mathrm{kg}$ a las semanas $0,2,6$ y luego cada 8 a 10 semanas en infusión intravenosa por un período de entre 2 y $3 \mathrm{~h}$. En caso de refractariedad se disminuyó el intervalo entre las dosis a 4 semanas.

Se registró la respuesta clínica tanto a nivel ocular como extraocular en controles periódicos cada 4 semanas.

Paciente 1. Hombre de 26 años con antecedentes de úlceras orales, genitales y foliculitis a repetición desde 1993. En 2001 presentó un accidente vascular isquémico de troncoencéfalo. Consultó en julio de 2003 por visión borrosa y disminución de la agudeza visual. El examen oftalmológico demostró una panuveítis bilateral con edema macular mayor en ojo derecho (OD). La agudeza visual (AV) era de 0,05 en el OD y de 0,2 en el ojo izquierdo (OI). Se indicó 3 pulsos de $1 \mathrm{~g}$ de metil prednisolona por pulso, seguido de prednisona 1 $\mathrm{mg} / \mathrm{kg} /$ día sin experimentar mejoría a los 7 días, por lo que se decidió iniciar tratamiento con infliximab.

Paciente 2. Mujer de 24 años que en agosto de 2004 comenzó con eritema nodoso de ambas extremidades inferiores. Tres semanas después se agregaron múltiples lesiones ulceradas dolorosas (al menos 15) en la mucosa oral y dos lesiones ulceradas en la mucosa vaginal. Consultó el 6 de octubre de 2004 por visión borrosa. El examen oftalmológico mostró Tyndall celular $(+++)$ en cámara anterior en ojo derecho e izquierdo (ODI), sinequias posteriores en OI, presencia de células $(+)$ en el vítreo anterior en ambos ojos. El fondo

Tabla 1. C aracterísticas demográficas de los pacientes incluidos en la serie

\begin{tabular}{|lll|}
\hline & Mujeres & Hombres \\
\hline Número & 3 & 2 \\
Rango de edad (años) & $24-49$ & $26-40$ \\
Promedio de edad (años) \pm DE & $34,66 \pm 12,9$ & $33 \pm 9,8$ \\
\hline
\end{tabular}


de ojo mostró trombosis de rama venosa, exudados algodonosos retinales a nasal y vasculitis moderada en OD. El OI mostraba trombosis de vena central, infiltrados retinales múltiples y severa vasculitis (Figura 1a). La angiofluoroesceinografía reveló además la presencia de zonas de isquemia retinal en OI (Figura 1b). La agudeza visual era de 0,6 en OD y de 0,15 en OI. Se encontraba sin tratamiento al momento de iniciar el infliximab.

Paciente 3. Mujer de 31 años que en mayo de 2000 comenzó con un síndrome febril no infeccioso con adenopatías cervicales de 1 mes de duración que remitió espontáneamente. En julio del mismo año presentó una vasculitis retinal. Refería el antece-

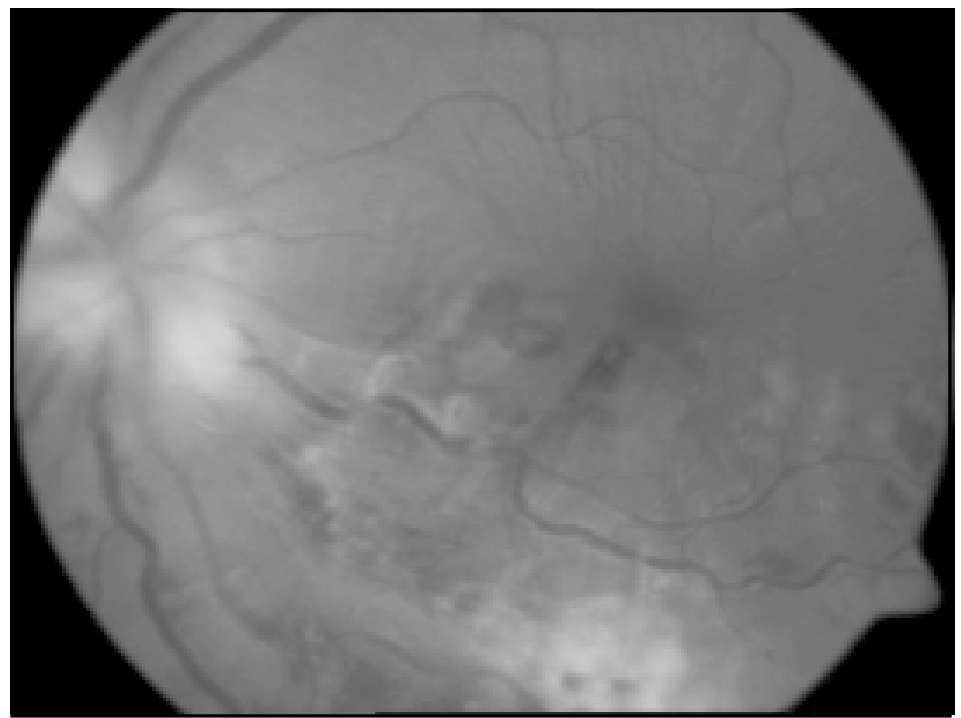

Figura 1a. Fondo de ojo de la paciente 2 al momento de iniciar terapia con infliximab. Se observa trombosis de vena central de la retina, numerosas hemorragias retinales en polo posterior y exudados algodonosos en relación a arcada temporal inferior.

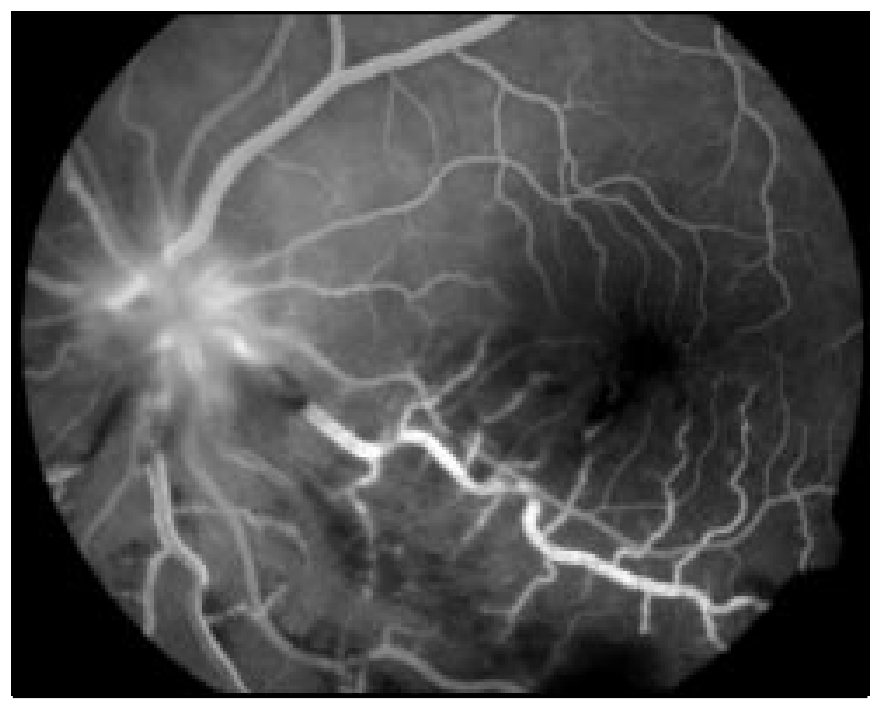

Figura 1b. Angiofluoroesceinografía de la paciente 2 al momento de iniciar terapia con infliximab. Se observa trombosis de vena central de la retina, hemorragias retinales, vasculitis arterial e isquemia retinal penipapilar inferior. 
dente de úlceras orales a repetición y úlceras genitales en dos oportunidades. Se solicitó HLA B51, cuyo resultado fue (+). Recibió tratamiento con metil prednisolona oral en dosis $64 \mathrm{mg} /$ día y ciclosporina A en dosis de 150 mg/día. Evolucionó satisfactoriamente y se redujeron los fármacos en forma progresiva hasta su suspensión en junio de 2003. En octubre de 2004 presentó nueva crisis ocular por lo que se inició tratamiento con prednisona en dosis de $60 \mathrm{mg} /$ día. Al mes de tratamiento se agregó disminución de la agudeza visual $(\mathrm{OD}=$ 0,6 y $\mathrm{OI}=0,2$ ) por lo que la paciente fue derivada a nuestro centro. Al examen oftalmológico el OD se encontraba normal. OI con células escasas en cámara anterior y en vítreo. El fondo de ojo en OI mostraba palidez papilar leve a temporal y sospecha de edema macular.

Paciente 4. Mujer de 49 años con historia clínica que data de 1996 cuando presentó una vasculitis de troncoencéfalo. En los meses y años siguientes se agregaron úlceras orales a repetición, eritema nodoso, foliculitis y un episodio de vasculitis retinal y panuveítis en $\mathrm{OD}$, con recurrencias sistémicas de la enfermedad en forma periódica con brotes de 2 a 3 meses de duración e intervalos libres variables de aproximadamente 6 meses. Consultó por un brote de panuveítis en OI en octubre de 2002, con agudeza visual de 0,8 en OD y cuenta dedos en OI. En ese momento la paciente se encontraba recibiendo prednisona 20 $\mathrm{mg} /$ día, azatioprina $100 \mathrm{mg} /$ día y talidomida en dosis de 200 mg/día.

Paciente 5. Hombre de 40 años que en diciembre de 2002 presentó úlceras orales recurrentes y eritema nodoso en extremidades inferiores. En febrero de 2003 se asoció un cuadro de uveítis anterior aguda y maculopatía en OD tratado con esteroides locales, obteniéndose regresión parcial del cuadro. Consultó médico internista quien planteó un SB e inició tratamiento con metil prednisolona $8 \mathrm{mg} /$ día, metotrexato (MTX) en dosis de $15 \mathrm{mg} / \mathrm{semanal}$ y pentoxifilina $400 \mathrm{mg} /$ día. Permaneció estable hasta abril de mismo año en que presentó un nuevo brote de uveítis, esta vez bilateral por lo que se agregaron esteroides locales que lograron controlar la crisis. Evolucionó en forma estable con metil prednisolona y MTX en igual dosis hasta noviembre de 2003 en que presentó nueva crisis de úlceras orales y genitales, compromiso del estado general, reactivación de la uveítis, piodermitis, eritema nodoso y artritis de ambos tobillos. En el examen ocular se constató un Tyndall (+) en OD y (+) en OI. Opacidades vítreas en OD con edema macular. La agudeza visual era de 0,4 en OD y de 1,0 en OI.

\section{Resultados}

El paciente 1 inició el tratamiento con infliximab y prednisona $1 \mathrm{mg} / \mathrm{kg} /$ día el 11 de julio de 2003. Se observó una respuesta clínica a las 48 h de la administración del infliximab y una franca mejoría al cabo de 2 semanas de la primera dosis. La agudeza visual mejoró a 0,5 en OD y 0,8 en OI. No de detectó inflamación a la biomicroscopia. Durante las siguientes 4 semanas la agudeza visual continuó mejorando a 0,8 parcial en OD y a 1,0 en OI, logrando suspender la prednisona a la sexta semana de tratamiento. Al cabo de la tercera dosis de infliximab debió suspender el tratamiento por realización de uretroplastia.

Permaneció inactivo del SB hasta el 20 de marzo de 2004 en que presentó una recaída del compromiso ocular, diagnosticándose una panuveítis bilateral y pesquisándose una disminución de la agudeza visual a 0,5 en el OI. Se reinició tratamiento con infliximab logrando controlar la inflamación intraocular y recuperando la visión en ambos ojos a 1,0 al mes de tratamiento. A la fecha se encuentra recibiendo infliximab cada 8 semanas. Su visión es normal y presenta mínima inflamación residual de la retina periférica en OI.

La paciente 2 inició infliximab el 8 de octubre de 2004. Se detectó una mejoría de la agudeza visual desde 0,6 a 1,0 en OD y desde 0,15 a 1,0 en OI a las 4 semanas de iniciado el tratamiento. El fondo de ojo de esa fecha mostró solamente fístulas venosas peripapilares en la arcada temporal inferior del OI (Figura 2a). La angiofluoroesceinografía del OI reveló resolución completa de la trombosis de la vena central de la retina, ausencia de vasculitis, persistiendo sólo hemorragias retinales escasas y pequeña fístula venosa peripapilar en arcada temporal inferior (Figura $2 b$ ). A la fecha se encuentra recibiendo infliximab cada 8 semanas sin presentar inflamación intraocular ni síntomas sistémicos. 


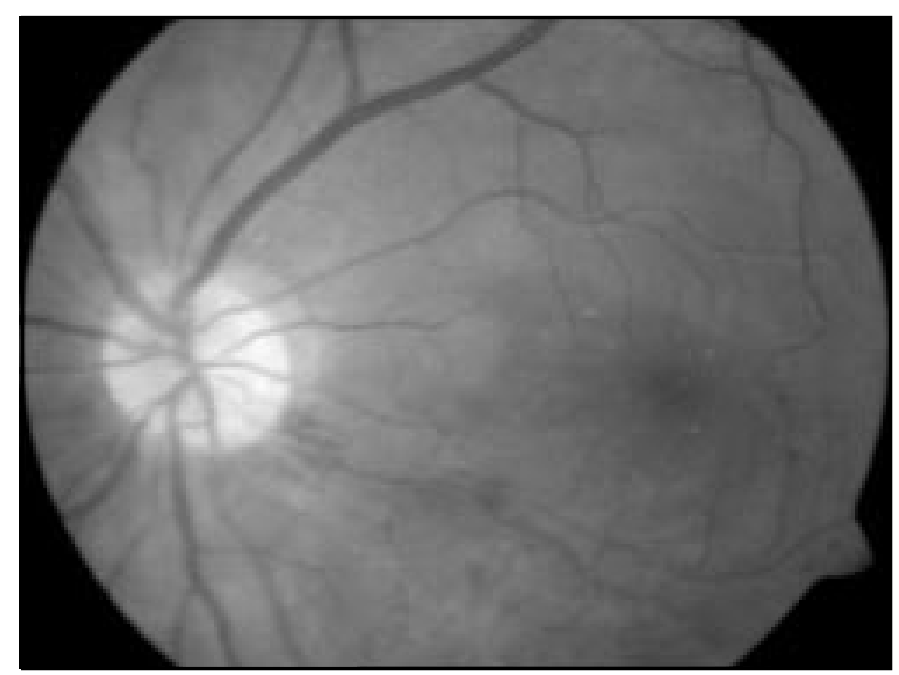

Figura 2a. Fondo de ojo de la paciente 2 a las cuatro semanas de iniciado el tratamiento con infliximab. Se observan escasas hemorragias retinales y pequeña fístula venosa peripapilar.

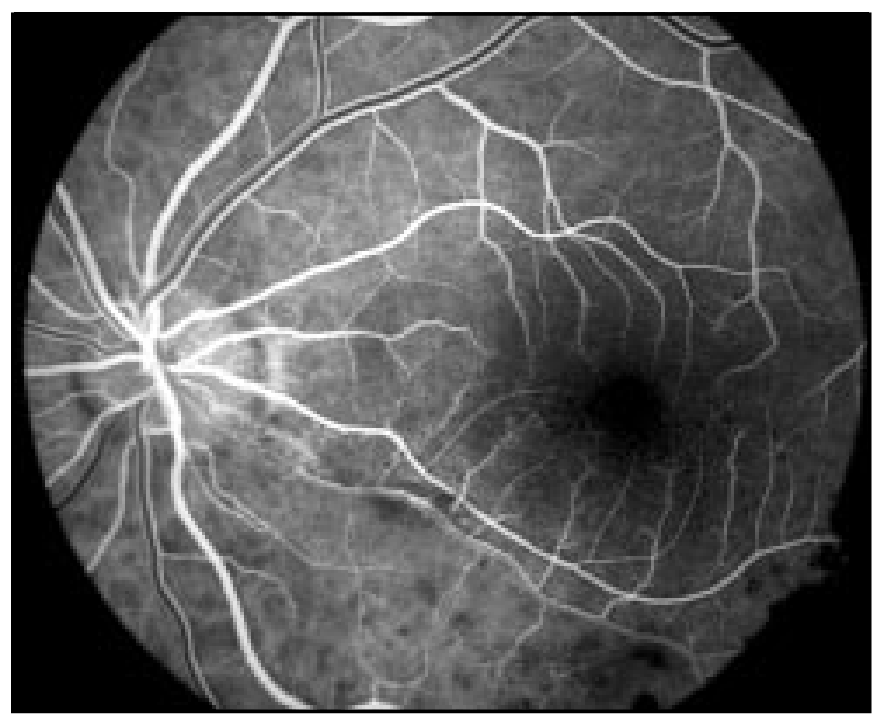

Figura 2b. Angiofluoroesceinografía de la paciente 2 a las cuatro semanas de iniciado el tratamiento con infliximab. Se observa sólo pequeña fístula venosa peripapilar y escasas hemorragias retinales.

La paciente 3 inició el infliximab el 15 de octubre de 2004. A los 10 días después de la primera infusión se apreció una mejoría de la agudeza visual en OD desde 0,6 a 1,0 y en OI desde 0,2 a 0,7 . La biomicroscopia era normal en ambos ojos. El fondo de ojo era normal en OD y mostraba una hemorragia retinal periférica a temporal en el OI. A la fecha la paciente se encuentra recibiendo infliximab cada 10 semanas y prednisona $5 \mathrm{mg} /$ día. La agudeza visual es 1,0 en ambos ojos y no hay inflamación ocular ni síntomas sistémicos. 
La paciente 4 inició infliximab en octubre de 2002 con recuperación de la visión en OI desde «cuenta dedos»a 0,6 a las tres semanas y 0,8 a las seis semanas. A la fecha se mantiene inactiva en terapia con infliximab cada 10 semanas.

El paciente 5 inició tratamiento con infliximab el 18 de noviembre de 2003 y se mantiene la metil prednisolona en dosis de $8 \mathrm{mg} /$ día y el MTX en 15 $\mathrm{mg} /$ semanal. Al mes de iniciada la terapia desaparecieron completamente las úlceras mucosas y el compromiso cutáneo, persistiendo la artritis de tobillos. La VOD mejoró de 0,4 a 0,6 y se mantuvo en 1,0 en el OI. El Tyndall era (-) en ambos ojos. Debido a la refractariedad de la artritis debió mantenerse con infusiones mensuales de infliximab, logrando inactivar la artritis al cabo de la cuarta dosis. Recibió un total de 5 infusiones al cabo de las cuales el paciente discontinuó el control en marzo de 2004. Continuó en tratamiento con metil prednisolona y MTX hasta junio de 2004 en que consultó por una nueva crisis de úlceras mucosas, poliartritis y uveítis por lo que se reinició el tratamiento con infliximab cada 4 semanas, logrando controlar el cuadro de uveítis después de la tercera dosis de infliximab y la artritis después de la sexta dosis del fármaco.

La Tabla 2 muestra un resumen de la respuesta al tratamiento con infliximab en los 5 pacientes.

Efectos colaterales. No se detectaron efectos adversos mayores como la aparición de tuberculosis, infecciones respiratorias graves, celulitis o pielonefritis aguda en los pacientes tratados.

\section{DisCUSIÓN}

El SB es la principal causa de ceguera en países como Japón y Turquía. Si bien no existen estudios de prevalencia en nuestro país, el diagnóstico de esta entidad se realiza con baja frecuencia en la práctica clínica en nuestro medio. Lo anterior puede explicarse por la participación de factores genéticos y por un subdiagnóstico del cuadro. Sin embargo, se estima que $10 \%$ de los pacientes con SB son refractarios a los esteroides e inmunosupresores.

En este estudio se incluyeron pacientes portadores de SB grave. Todos ellos presentaban severo compromiso ocular y ha sido reportado que el TNF- $\alpha$ es un importante mediador de la inflamación intraocular ${ }^{12}$. Hemos comunicado recientemente que pacientes portadores de SB activo presentan altos títulos de anticuerpos de clase IgG deglicosilada anti-acido lipoteicoico (LTA) (un antígeno de origen estreptocócico) y que estos anticuerpos activan complemento in vitro. Del mismo modo los monocitos periféricos de estos pacientes producen gran cantidad de interleuquina-8 (IL-8) frente al estímulo con LTA ${ }^{13}$. A su vez ha sido reportado que el LTA es capaz de inducir la producción de TNF- $\alpha$, IL-1ß, IL-6 y de IL-8 en monocitos humanos in vitro ${ }^{14}$. Es decir es posible postular que antígenos ambientales podrían estimular la producción de citoquinas proinflamatorias en células mononucleares de pacientes con SB lo que contribuiría a explicar la reactivación de la enfermedad. Estas observacio-

Tabla 2. C aracterísticas del compromiso ocular, respuesta al infliximab y efectos adversos graves

\begin{tabular}{|ccccc|}
\hline Paciente & $\begin{array}{c}\text { Agudeza visual } \\
\text { pretratamiento }\end{array}$ & $\begin{array}{c}\text { Agudeza visual } \\
\text { luego de 3 dosis de } \\
\text { infliximab }\end{array}$ & $\begin{array}{c}\text { Control de la } \\
\text { inflamación } \\
\text { ocular al año de } \\
\text { seguimiento }\end{array}$ & $\begin{array}{c}\text { Efectos adversos } \\
\text { graves a los 6 } \\
\text { meses }\end{array}$ \\
\hline 1 & OD 0,05 OI 0,2 & OD 0,8 OI 1,0 & + & - \\
2 & OD 0,6 OI 0,15 & OD 1,0 OI 1,0 & + & - \\
3 & OD 0,6 OI 0,2 & OD 1,0 OI 1,0 & + & - \\
4 & OD 0,8 OI CD & OD 0,8 OI 1,0 & + & - \\
5 & OD 0,4 OI 1,0 & OD 0,6 OI 1,0 & + & - \\
\hline
\end{tabular}

$\mathrm{CD}=$ Cuenta Dedos, OD= ojo derecho, $\mathrm{OI}=$ ojo izquierdo. 
nes sobre la patogenia del SB son concordantes con la eficacia del tratamiento anti-TNF- $\alpha$ en esta enfermedad.

El infliximab administrado en dosis de entre 3 a $5 \mathrm{mg} / \mathrm{kg}$ demostró ser eficaz en el control de los síntomas y signos oculares en nuestros pacientes. Nuestros resultados son concordantes con los de otros autores, que reportan un dramático efecto del infliximab en el control del compromiso ocular en el SB ${ }^{10,11,15}$.

En relación al compromiso extraocular, el infliximab parece ser igualmente eficaz ya que tanto las úlceras mucosas como la piodermitis desaparecieron con el tratamiento, tal como ha sido reportado por otros autores ${ }^{16}$. Un problema aparte es la artritis. El paciente 5 presentaba una severa artritis la que fue de difícil control y obligó a acortar el intervalo de administración del fármaco. Este paciente recibió una dosis de $5 \mathrm{mg} / \mathrm{kg}$ que es superior a la utilizada en la artritis reumatoidea y aun así requirió de administraciones más frecuentes que las reportadas en la artritis reumatoidea. Lo anterior enfatiza la necesidad de identificar la dosis terapéutica eficaz de los fármacos anti TNF- $\alpha$ en cada patología por separado y no extrapolar resultados obtenidos en otras patologías inflamatorias que también producen sinovitis. Del mismo modo la eficacia del infliximab en UR asociada al SB no puede ser extrapolada a pacientes con otros tipos de uveítis. El diagnóstico de uveítis involucra una serie de patologías

\section{REFERENCIAS}

1. Kaklamani VG, Vaiopoulos G, Kaklamanis G. Behçet's disease. Semin Arthritis Rheum 1998; 27: 197-217.

2. Sakane T, Takeno M, Suzuki N, Inaba G. Behçet's disease. N Engl J Med 1999; 341: 1284-91.

3. Nussenblatt RB. Uveitis in Behçet's disease. Int Rev Immunol 1997; 14: 67-79.

4. Nakamura S, Yamakawa $T$, Sugita M, Kijima $M$, ISHIOKA $M$, TANAKA $S$ ET aL. The role of tumor necrosis factor-alpha in the induction of experimental autoimmune uveoretinitis in mice. Invest Ophthalmol Vis Sci 1994; 35: 3884-9. heterogéneas, muchas de las cuales pueden completar criterios diagnósticos meses o años después del primer brote de inflamación ocular y los fármacos anti TNF- $\alpha$ no son necesariamente eficaces en todas ellas.

$\mathrm{Si}$ bien, los reportes publicados sugieren que el infliximab parece ser bien tolerado en el SB, nos parece importante destacar que el número de pacientes tratados a la fecha es muy pequeño como para poder detectar efectos colaterales significativos. Ha sido reportado que el TNF- $\alpha$ posee un efecto antitrombótico ${ }^{17}$ y existen reportes preliminares que sugieren que el infliximab podría aumentar la incidencia de fenómenos tromboembólicos durante el tratamiento anti$\mathrm{TNF}^{18}{ }^{18}$. Una de las manifestaciones clínicas del SB es la trombosis arterial y venosa, por lo que estos pacientes deberían ser observados acuciosamente durante el tratamiento para descartar la aparición de un fenómeno trombótico.

En conclusión, el tratamiento anti-TNF- $\alpha$ parece ser una alternativa eficaz en el manejo del SB severo y/o refractario a terapia esteroidal e inmunosupresora. El efecto benéfico de este tratamiento apoya la hipótesis que el TNF- $\alpha$ es un mediador importante en el patogenia del SB. Nuestros resultados así como los de otros autores enfatizan la necesidad de diseñar estudios prospectivos randomizados que evalúen la eficacia de la terapia anti-TNF- $\alpha$ en los pacientes con SB. 
8. Lipsky PE, van der Heijde DM, St Clair EW, Furst DE, BReEDVeld FC, KaLden JR et al. Infliximab and methotrexate in the treatment of rheumatoid arthritis. N Engl J Med 2000; 343: 1594-602.

9. Cuchacovich M, Ferreira L, Aliste M, Soto L, Cuenca J, Cruzat A et al. TNF- $\alpha$ levels and influence of -308 TNF- $\alpha$ promoter polymorphism on the responsiveness to infliximab in patients with rheumatoid arthritis. Scand J Rheumatol 2004; 33: 228-32.

10. Sfikakis PP, Theodossiadis PG, Katsiari CG, KakiaMANIS P, MaRKOMICHELAKIS NN. Effect of infliximab on sight-threatening panuveitis in Behçet's disease. Lancet 2001; 58: 295-6.

11. Ohno S, Satoshi N, Hori S, Shimakawa M, Kawashima $\mathrm{H}$, MochizUKi M ET AL. Efficacy, safety, and pharmacokinetics of multiple administration of infliximab in Behçet's disease with refractory uveoretinitis. J Rheumatol 2004; 31: 1362-8.

12. Lacomba SM, Martin MC, Galardo Galera JM, Gómez Vidal MA, CoLantes Estévez E, Ramírez Chamond R et aL. Aqueous humor and serum tumor necrosis factor-alpha in clinical uveitis. Ophthalmic Res 2001; 33: 251-5.
13. Cuchacovich M, Merino G, Yamamoto J, Viliarroel F, SAAVEDRA T, Jofré $S$ et al. Behçet's disease patients present high levels of deglycosylated anti-lipoteichoic acid IgG and high IL-8 production after lipoteichoic acid stimulation. Clin Exp Rheumatol 2005; 23: S27-S34.

14. Mancuso G, Tomasello F, Ofek I, Teti G. AntiLipoteichoic acid antibodies enhance release of cytokines by monocytes sensitized with lipoteichoic acid. Infect Immun 1994; 62: 1470-3.

15. Muñoz-Fernández S, Hidalgo V, Fernández-Melón J, SCHLiNCKer A, Martín Mola E. Effect of infliximab on threatening panuveitis in Behçet's disease. Lancet 2001; 358: 1644.

16. Haugeberg G, Velken M, Johnsen V. Successful treatment of genital ulcers with infliximab in Behçet's disease. Ann Rheum Dis 2004; 63: 744-5.

17. Cambien B, Bergmeier W, Saffaripour S, Mitchel HA, WAGNER DD. Antithrombotic activity of TNFalpha. J Clin Invest 2003; 112: 1589-96.

18. RosenBAUM JT. Blind Insight: Eyeing anti-tumor necrosis factor treatment in uveitis associated with Behçet's Disease. J Rheumatol 2004; 31: 1241-3. 